\title{
Global Stability and Optimal Control of Dengue with Two Coexisting Virus Serotypes
}

\author{
${ }^{1}$ Afeez Abidemi*, ${ }^{2}$ Rohanin Ahmad and ${ }^{3}$ Nur Arina Bazilah Aziz \\ 1,2,3 Department of Mathematical Sciences, Universiti Teknologi Malaysia \\ 81310 Johor Bahru, Johor, Malaysia \\ ${ }^{1}$ Department of Mathematical Sciences, Federal University of Technology, Akure \\ P.M.B. 704, Ondo State, Nigeria \\ *Corresponding author: aabidemi@futa.edu.ng
}

Article history

Received: 1o November 2019

Received in revised form: 19 November 2019

Accepted: 23 December 2019

Published online: 31 December 2019

\begin{abstract}
This study presents a two-strain deterministic model which incorporates Dengvaxia vaccine and insecticide (adulticide) control strategies to forecast the dynamics of transmission and control of dengue in Madeira Island if there is a new outbreak with a different virus serotypes after the first outbreak in 2012. We construct suitable Lyapunov functions to investigate the global stability of the disease-free and boundary equilibrium points. Qualitative analysis of the model which incorporates time-varying controls with the specific goal of minimizing dengue disease transmission and the costs related to the control implementation by employing the optimal control theory is carried out. Three strategies, namely the use of Dengvaxia vaccine only, application of adulticide only, and the combination of Dengvaxia vaccine and adulticide are considered for the controls implementation. The necessary conditions are derived for the optimal control of dengue. We examine the impacts of the control strategies on the dynamics of infected humans and mosquito population by simulating the optimality system. The disease-free equilibrium is found to be globally asymptotically stable whenever the basic reproduction numbers associated with virus serotypes 1 and $j(j \in\{2,3,4\})$, respectively, satisfy $\mathcal{R}_{01}, \mathcal{R}_{0 j} \leq 1$, and the boundary equilibrium is globally asymptotically stable when the related $\mathcal{R}_{0 i}(i=1, j)$ is above one. It is shown that the strategy based on the combination of Dengvaxia vaccine and adulticide helps in an effective control of dengue spread in the Island.
\end{abstract}

Keywords Dengue model; Lyapunov function; optimal control; stability analysis.

Mathematics Subject Classification 49J15, 92B05, 93A30.

\section{Introduction}

Dengue fever is a mosquito-borne disease caused by the dengue virus of the family Flaviviridae, which has four distinguished but closely related serotypes (or strains) identified as DEN-1, 2, 3, 4 
$[1,2]$. The disease is transmitted to human by the bites of its infected primary and secondary vectors Aedes aegypti and Aedes albopictus, respectively [2,3]. Of the four dengue virus serotypes that affect humans, DEN-2 and DEN-3 have potential to cause more severe dengue than DEN-1 and DEN-4 [4]. Human recovery from an infection by one dengue virus strain confers lifelong immunity to the strain, but only temporary cross-immunity to the rest [2], which increases the susceptibility of such individual to the other three virus serotypes [2]. Epidemiological studies of dengue revealed that dengue haemorrhagic fever (DHF) or dengue shock syndrome is more likely to occur in secondary infection $[5,6]$.

Up to date, there is no perfect dengue vaccine. Live attenuated Dengvaxia vaccine was licensed for the disease in December 2015 [3]. It is evident from studies that the vaccine is efficacious and safe in seropositive individuals (that is, persons confirmed to have had dengue infection before vaccination) and exposes the seronegative individuals (i.e, persons with first dengue infection after vaccination) to a severe risk of the infection based on the outcome of clinical trials. As a result, the World Health Organization (WHO) recommended pre-vaccination screening strategy, which aims at vaccinating seropositive individuals [3]. Meanwhile, effective control strategy of dengue focuses on its vector, which depends on the reduction of mosquito population. This includes administering larvicide at mosquito breeding sites, open space spraying using insecticide, removal of artificial mosquito breeding sites, and personal protection against mosquito bites [3].

Compartmental models have been used to describe the transmission dynamics of dengue between the interacting host and vector populations. The models have helped to facilitate the comprehension of mechanisms involved in the dynamic of dengue disease transmission. Single strain models have been used to examine the transmission dynamics of dengue $[7,8]$. Others have examined the impact of vaccination on the transmission dynamics of dengue [9], investigate the efficacy of vector control strategies on dengue spread [10,11], and assessed the impact of combining vector controls and imperfect vaccine on the spread of dengue [12]. However, two-strain deterministic models have been used to describe the transmission dynamics of the coexistence of two dengue virus serotypes [1,13-15] and examine the impact of control strategies on the transmission of dengue $[4,16,17]$. Mishra and Gakkhar [18] used a two-strain mathematical model to assess the impacts of awareness and vector controls on the dynamics of transmission of dengue.

In another developments, investigation of optimal strategies for preventing and controlling dengue disease transmission has been carried out by employing optimal control (OC) theory. For instance, Agusto and Khan [19] and Pongsumpun et al. [20] derived the optimal levels of vaccination and insecticide for dengue disease prevention and control. Also, optimal insecticide control was determined in Rodrigues et al. [21]. In another study, Buonomo and Marca [22] formulated an OC problem governed by a system of ordinary differential equations (ODEs) to obtain the optimal bed net and insecticide controls. However, non of the authors that incorporated vaccination into their models took into consideration the suggestion by WHO on the use of Dengvaxia vaccine. Hence, a reliable two-strain deterministic model is essential to better comprehend the mechanism of dengue disease spread and control using Dengvaxia vaccine and adulticide.

Our interest in this work is to propose an OC framework based on a two-strain dengue model which does not only consider the open field spraying of adulticide, but also the vaccination of seropositive individuals with the consideration of the recommendation by WHO [3] on the use 
of Dengvaxia. The underlying idea is to examine the effect of an integrated form of the control strategy (use of adulticide only) adopted in Rocha et al. [4] on the dynamical system of dengue disease transmission with coexistence of two virus serotypes. More precisely, we use information from the 2012 dengue outbreak in Madeira Island, Portugal as reported by Rocha et al. [4] to forecast the scenarios of coexistence of two virus strains in the Island. Three strategies, namely use of Dengvaxia vaccine only, use of adulticide only, and combination of Dengvaxia vaccine and adulticide, are adopted for implementing the controls.

The rest of this paper is organized as follows. In Section 2, a two-strain dengue model which incorporates two control parameters $u_{1}$ and $u_{2}$ accounting for Dengvaxia vaccine and adulticide, respectively, is proposed. Section 3 presents the analysis of the model. In Section 4, formulation of OC problem is taken up by considering the controls $u_{1}$ and $u_{2}$ as timedependent variables. We employ Pontryagin's maximum principle (PMP) in order to examine the necessary conditions for the optimal strategies for the control of dengue disease spread, and numerical simulations are conducted on the resulting optimality system. Results and discussion are taken up in Section 5. Conclusion is finally drawn in Section 6.

\section{The Model}

The two-strain deterministic model formulated in this paper is based on the single-strain dengue model proposed in Rodrigues et al. [21] and the consideration of the two-strain models presented in James [13] and Rocha et al. [4]. The model describes the interactions between human and mosquito populations. The model subgroups human population into eight epidemiological states:

$H_{S}(t)$ - Susceptible individuals who can contract both serotypes 1 and $j$;

$H_{I_{1}}(t)$ - Infected individuals with serotype-1;

$H_{I_{j}}(t)$ - Infected individuals with serotype- $j$;

$H_{S_{1 j}}(t)$ - Recovered individuals from serotype-1 and susceptible to serotype- $j$;

$H_{S_{j 1}}(t)$ - Recovered individuals from serotype- $j$ and susceptible to serotype-1;

$H_{I_{1 j}}(t)$ - Infected individuals by serotype- $j$ after recovery from infection by serotype-1;

$H_{I_{j 1}}(t)$ - Infected individuals by serotype-1 after recovery from infection by serotype- $j$; and

$H_{R}(t)-$ Recovered individuals from secondary infection.

The mosquito population, which includes only female mosquitoes, is compartmentalized into three:

$M_{S}(t)$ - Susceptible mosquitoes that can contract both serotypes 1 and $j$;

$M_{I_{1}}(t)$ - Infected mosquitoes by serotype-1; and

$M_{I_{j}}(t)$ - Infected mosquitoes by serotype- $j$. 
The 2012 first dengue outbreak in Madeira Island was caused by DEN-1 [4] and there may be a new disease outbreak, if anyone of the other three serotypes surfaces in the Island. Therefore, we are not specific about the virus serotype to be responsible for the new outbreak. Then, we use the same idea adopted in Rocha et al. [4] by letting the index $j$ denotes the second virus serotype, which is a member of the set $\{2,3,4\}$ representing DEN-2, DEN-3 and DEN-4, respectively. We retain the same notation henceforth unless otherwise specified.

In the formulation of our model, variable population sizes for both human and mosquito populations are considered. This is due to the consideration of the value different from zero for the disease-induced death rate $\left(\mu_{D} \neq 0\right)$, which represents the probability of death from DHF, and increasing mortality rate of mosquito at a rate $u_{2}(t)$. Therefore, the total human and mosquito populations at any time $t$ are given by Equations (1) and (2), respectively, as

$$
H_{N}=H_{S}(t)+H_{I_{1}}(t)+H_{I_{j}}(t)+H_{S_{1 j}}(t)+H_{S_{j 1}}(t)+H_{I_{1 j}}(t)+H_{I_{j 1}}(t)+H_{R}(t)
$$

and

$$
M_{N}=M_{S}(t)+M_{I_{1}}(t)+M_{I_{j}}(t)=m H_{N}
$$

for some constant $m[21]$.

Also, two control measures (insecticide and vaccination) are considered for interventions. Control parameter $u_{1}$, which represents the rate of vaccinating seropositive individuals, is incorporated into the model in line with WHO directives on the use of the licensed live attenuated Dengvaxia vaccine on seropositive individuals [3]. Also, the model includes control parameter $u_{2}$ which accounts for adulticide control. One of the underlying ideas of this study is the fact that secondary infection increases the chance of developing DHF which can be justified by Antibody-Dependent Enhancement (ADE) phenomenon [4].

Formulation of the proposed model is made under the following assumptions:

i. There is no state to describe resistant mosquitoes because of their short lifespan;

ii. There is no state equation for secondary infected mosquitoes. Thus a mosquito, once infected, remains infectious for the rest of its lifetime [23];

iii. There is no immigration or emigration;

iv. It is assumed that humans and mosquitoes are born susceptible;

v. Human and mosquito populations are homogeneously mixing so that vector have an equal probability to bite any individual;

vi. Both vector and host can transmit the virus only during the infectious state;

vii. There is no vertical transmission of the virus in the vector and host; and

viii. Humans cannot be infected by more than one virus serotype at the same time.

Hence, the dynamics of vector-host interactions in the presence of two dengue virus strains is described by the system of ODEs for model 3 as shown in Equation (3). 


$$
\begin{aligned}
& \frac{d H_{S}(t)}{d t}=\mu_{H} H_{N}(t)-\left[B \beta_{M H_{1}} \frac{M_{I_{1}}(t)}{H_{N}(t)}+B \beta_{M H_{j}} \frac{M_{I_{j}}(t)}{H_{N}(t)}+\mu_{H}\right] H_{S}(t), \\
& \frac{d H_{I_{1}}(t)}{d t}=B \beta_{M H_{1}} \frac{M_{I_{1}}(t)}{H_{N}(t)} H_{S}(t)-\theta_{H_{1}} H_{I_{1}}(t)-\mu_{H} H_{I_{1}}(t), \\
& \frac{d H_{I_{j}}(t)}{d t}=B \beta_{M H_{j}} \frac{M_{I_{j}}(t)}{H_{N}(t)} H_{S}-\theta_{H_{j}} H_{I_{j}}(t)-\mu_{H} H_{I_{j}}(t), \\
& \frac{d H_{S_{1 j}}(t)}{d t}=\theta_{H_{1}} H_{I_{1}}(t)-\sigma_{j} B \beta_{M H_{j}} \frac{M_{I_{j}}(t)}{H_{N}(t)} H_{S_{1 j}}(t)-\mu_{H} H_{S_{1 j}}(t)-u_{1}(t) H_{S_{1 j}}(t), \\
& \frac{d H_{S_{j 1}}(t)}{d t}=\theta_{H_{j}} H_{I_{j}}(t)-\sigma_{1} B \beta_{M H_{1}} \frac{M_{I_{1}}(t)}{H_{N}(t)} H_{S_{j 1}}(t)-\mu_{H} H_{S_{j 1}}(t)-u_{1}(t) H_{S_{j 1}}(t), \\
& \frac{d H_{I_{1 j}}(t)}{d t}=\sigma_{j} B \beta_{M H_{j}} \frac{M_{I_{j}}(t)}{H_{N}(t)} H_{S_{1 j}}(t)-\theta_{H_{j}} H_{I_{1 j}}(t)-\mu_{H} H_{I_{1 j}}(t)-\mu_{D} H_{I_{1 j}}(t), \\
& \frac{d H_{I_{j 1}}(t)}{d t}=\sigma_{1} B \beta_{M H_{1}} \frac{M_{I_{1}}(t)}{H_{N}(t)} H_{S_{j 1}}(t)-\theta_{H_{1}} H_{I_{j 1}}(t)-\mu_{H} H_{I_{j 1}}(t)-\mu_{D} H_{I_{j 1}}(t), \\
& \frac{d H_{R}(t)}{d t}=\theta_{H_{1}} H_{I_{j 1}}(t)+\theta_{H_{j}} H_{I_{1 j}}(t)+u_{1}(t) H_{S_{1 j}}(t)+u_{1}(t) H_{S_{j 1}}(t)-\mu_{H} H_{R}(t), \\
& \frac{d M_{S}(t)}{d t}=\mu_{M} M_{N}(t)-\left[B \beta_{H M_{1}} \frac{\left(H_{I_{1}}(t)+H_{I_{j 1}}(t)\right)}{H_{N}(t)}+B \beta_{H M j} \frac{\left(H_{I_{j}}(t)+H_{I_{1 j}}(t)\right)}{H_{N}(t)}\right] M_{S}(t) \\
& -\left(\mu_{M}+u_{2}(t)\right) M_{S}(t) \\
& \frac{d M_{I_{1}}(t)}{d t}=B \beta_{H M_{1}} \frac{\left(H_{I_{1}}(t)+H_{I_{j 1}}(t)\right)}{H_{N}(t)} M_{S}(t)-\mu_{M} M_{I_{1}}(t)-u_{2}(t) M_{I_{1}}(t), \\
& \frac{d M_{I_{j}}(t)}{d t}=B \beta_{H M_{j}} \frac{\left(H_{I_{j}}(t)+H_{I_{1 j}}(t)\right)}{H_{N}(t)} M_{S}(t)-\mu_{M} M_{I_{j}}(t)-u_{2}(t) M_{I_{j}}(t),
\end{aligned}
$$

subject to the initial conditions (ICs):

$$
\begin{gathered}
H_{S}(0)=H_{0 S}, \quad H_{I_{1}}(0)=H_{0 I_{1}}, \quad H_{I_{j}}(0)=H_{0 I_{j}}, \quad H_{S_{1 j}}(0)=H_{0 S_{1 j}}, \\
H_{S_{j 1}}(0)=H_{0 S_{j 1}}, \quad H_{I_{1 j}}(0)=H_{0 I_{1 j}}, \quad H_{I_{j 1}}(0)=H_{0 I_{j 1}}, \quad H_{R}(0)=H_{0 R}, \\
M_{S}(0)=M_{0 S}, \quad M_{I_{1}}(0)=M_{0 I_{1}}, \quad M_{I_{j}}(0)=M_{0 I_{j}} .
\end{gathered}
$$

Description of the parameters of Model (3) is presented in Table 1.

The 8th ODE in Equation (3) describes the population of recovered individuals in the dynamical system decouples and can be excluded from Model 3 based on the condition in Equation (1), and use

$$
H_{R}(t)=H_{N}(t)-H_{S}(t)-H_{I_{1}}(t)-H_{I_{j}}(t)-H_{S_{1 j}}(t)-H_{S_{j 1}}(t)-H_{I_{1 j}}(t)-H_{I_{j 1}}(t) .
$$


Table 1: Model Parameter Values and Their Description

\begin{tabular}{|c|c|c|c|c|}
\hline \multirow{2}{*}{ Parameter } & \multirow{2}{*}{ Description } & \multicolumn{2}{|c|}{ Value } & \multirow{2}{*}{ Source } \\
\hline & & Scenario 1 & Scenario 2 & \\
\hline$H_{N}$ & Total human population & 112000 & 112000 & {$[4,11]$} \\
\hline$M_{N}$ & Total mosquito population & $m \times H_{N}$ & $m \times H_{N}$ & \\
\hline$\sigma_{1}, \sigma_{j}$ & Susceptibility indexes for secondary infection by virus serotypes 1 and $j$, respectively & $1.1,1.1$ & $1.1,1.1$ & {$[4]$} \\
\hline$\beta_{M H_{1}}, \beta_{M H_{j}}$ & $\begin{array}{l}\text { Transmission probabilities of dengue virus from infected mosquito by serotypes } 1 \text { and } \\
j, \text { respectively, to human (per bite) }\end{array}$ & $0.25,0.25$ & $0.25,0.33$ & {$[4]$} \\
\hline$\mu_{H}$ & Per capita birth rate and natural death rate of humans (per day) & $\frac{1}{79 \times 365}$ & $\frac{1}{79 \times 365}$ & {$[4,11]$} \\
\hline$B$ & Average mosquito biting rate (per day) & $\frac{1}{3}$ & $\frac{1}{3}$ & {$[4,11]$} \\
\hline$\beta_{H M_{1}}, \beta_{H M_{j}}$ & $\begin{array}{l}\text { Transmission probabilities of dengue virus from infected human by serotypes } 1 \text { and } j, \\
\text { respectively, to mosquito (per bite) }\end{array}$ & $0.25,0.25$ & $0.25,0.33$ & {$[4]$} \\
\hline$\frac{1}{\mu_{M}}$ & Per capita death rate of mosquito (in days) & 15 & 15 & {$[4,11]$} \\
\hline$\frac{1}{\theta_{H_{1}}}, \frac{1}{\theta_{H_{j}}}$ & Average periods of infectiousness of virus strains 1 and $j$ in human (in days) & 7,5 & 7,9 & {$[4]$} \\
\hline$\mu_{D}$ & Dengue virus disease-induced death rate & 0.02 & 0.02 & {$[4]$} \\
\hline$m$ & Number of female mosquito per human & 6 & 6 & {$[10]$} \\
\hline
\end{tabular}


Then, the equivalent system for human and mosquito populations for model 5 is described in Equation (5).

$$
\begin{aligned}
& \frac{d H_{S}(t)}{d t}=\mu_{H} H_{N}(t)-\left[B \beta_{M H_{1}} \frac{M_{I_{1}}(t)}{H_{N}(t)}+B \beta_{M H_{j}} \frac{M_{I_{j}}(t)}{H_{N}(t)}+\mu_{H}\right] H_{S}(t), \\
& \frac{d H_{I_{1}}(t)}{d t}=B \beta_{M H_{1}} \frac{M_{I_{1}}(t)}{H_{N}(t)} H_{S}(t)-\theta_{H_{1}} H_{I_{1}}(t)-\mu_{H} H_{I_{1}}(t), \\
& \frac{d H_{I_{j}}(t)}{d t}=B \beta_{M H_{j}} \frac{M_{I_{j}}(t)}{H_{N}(t)} H_{S}-\theta_{H_{j}} H_{I_{j}}(t)-\mu_{H} H_{I_{j}}(t), \\
& \frac{d H_{S_{1 j}}(t)}{d t}=\theta_{H_{1}} H_{I_{1}}(t)-\sigma_{j} B \beta_{M H_{j}} \frac{M_{I_{j}}(t)}{H_{N}(t)} H_{S_{1 j}}(t)-\mu_{H} H_{S_{1 j}}(t)-u_{1}(t) H_{S_{1 j}}(t), \\
& \frac{d H_{S_{j 1}}(t)}{d t}=\theta_{H_{j}} H_{I_{j}}(t)-\sigma_{1} B \beta_{M H_{1}} \frac{M_{I_{1}}(t)}{H_{N}(t)} H_{S_{j 1}}(t)-\mu_{H} H_{S_{j 1}}(t)-u_{1}(t) H_{S_{j 1}}(t), \\
& \frac{d H_{I_{1 j}}(t)}{d t}=\sigma_{j} B \beta_{M H_{j}} \frac{M_{I_{j}}(t)}{H_{N}(t)} H_{S_{1 j}}(t)-\theta_{H_{j}} H_{I_{1 j}}(t)-\mu_{H} H_{I_{1 j}}(t)-\mu_{D} H_{I_{1 j}}(t), \\
& \frac{d H_{I_{j 1}}(t)}{d t}=\sigma_{1} B \beta_{M} H_{1} \frac{M_{I_{1}}(t)}{H_{N}(t)} H_{S_{j 1}}(t)-\theta_{H_{1}} H_{I_{j 1}}(t)-\mu_{H} H_{I_{j 1}}(t)-\mu_{D} H_{I_{j 1}}(t), \\
& \frac{d M_{S}(t)}{d t}=\mu_{M} M_{N}(t)-\left[B \beta_{H M_{1}} \frac{\left(H_{I_{1}}(t)+H_{I_{j 1}}(t)\right)}{H_{N}(t)}+B \beta_{H M j} \frac{\left(H_{I_{j}}(t)+H_{I_{1 j}}(t)\right)}{H_{N}(t)}\right] M_{S}(t) \\
&-\left(\mu_{M}+u_{2}(t)\right) M_{S}(t), \\
& \frac{d M_{I_{1}}(t)}{d t}= B \beta_{H M_{1}} \frac{\left(H_{I_{1}}(t)+H_{I_{j 1}}(t)\right)}{H_{N}(t)} M_{S}(t)-\mu_{M} M_{I_{1}}(t)-u_{2}(t) M_{I_{1}}(t), \\
& \frac{d M_{I_{j}}(t)}{d t}= B \beta_{H M_{j}} \frac{\left(H_{I_{j}}(t)+H_{I_{1 j}}(t)\right)}{H_{N}(t)} M_{S}(t)-\mu_{M} M_{I_{j}}(t)-u_{2}(t) M_{I_{j}}(t),
\end{aligned}
$$

with

$$
H_{S}(t)+H_{I_{1}}(t)+H_{I_{j}}(t)+H_{S_{1 j}}(t)+H_{S_{j 1}}(t)+H_{I_{1 j}}(t)+H_{I_{j 1}}(t) \leq H_{N}(t)
$$

and

$$
M_{N}(t)=M_{S}(t)+M_{I_{1}}(t)+M_{I_{j}}(t)=m H_{N}(t) .
$$

Since Model (5) is used to describe the interactions between host and vector, it is necessary to carry out some analyses of it.

\section{Analysis of the Model}

\subsection{Region of Biological Interest}

\section{Theorem 1 The region $\Gamma$ defined as}

$$
\begin{aligned}
\Gamma=\{ & \left(H_{S}(t), H_{I_{1}}(t), H_{I_{j}}(t), H_{S_{1 j}}(t), H_{S_{j 1}}(t), H_{I_{1 j}}(t), H_{I_{j 1}}(t), M_{S}(t), M_{I_{1}}(t), M_{I_{j}}(t)\right) \in \mathbb{R}_{+}^{10} \mid \\
& H_{S}(t) \leq H_{N}(t), H_{I_{1}}(t) \leq H_{N}(t), H_{I_{j}}(t) \leq H_{N}(t), H_{S_{1 j}}(t) \leq H_{N}(t), H_{S_{j 1}}(t) \leq H_{N}(t), \\
& H_{I_{1 j}}(t) \leq H_{N}(t), H_{I_{j 1}}(t) \leq H_{N}(t), M_{S}(t) \leq M_{N}(t), M_{I_{1}}(t) \leq M_{N}(t), M_{I_{j}}(t) \leq M_{N}(t), \\
& H_{S}(t)+H_{I_{1}}(t)+H_{I_{j}}(t)+H_{S_{1 j}}(t)+H_{S_{j 1}}(t)+H_{I_{1 j}}(t)+H_{I_{j 1}}(t) \leq H_{N}(t) \\
& \text { and } \left.M_{S}(t)+M_{I_{1}}(t)+M_{I_{j}}(t)=M_{N}(t)\right\}
\end{aligned}
$$

is positively invariant with respect to Model (5). 
Proof See Rodrigues et al. [10].

\section{$3.2 \quad$ Existence of Equilibria}

The equilibrium points related to Model (5) are attained when the differential equation describing each compartment is at steady state. By setting the right-hand side of each ODE of the system, Model (5), to zero, we identify four equilibrium points:

i. Disease-Free Equilibrium (DFE) obtained as

$$
\mathcal{E}_{0}=\left(H_{N}, 0,0,0,0,0,0, \frac{\mu_{M} M_{N}}{\left(\mu_{M}+u_{2}\right)}, 0,0\right) .
$$

ii. Two boundary equilibrium (BE) points $\mathcal{E}_{1}$ and $\mathcal{E}_{2}$, given by

$$
\mathcal{E}_{1}=\left(\left(H_{S}\right)_{1}^{*}, H_{I_{1}}^{*}, 0, H_{S_{1 j}}^{*}, 0,0,0,\left(M_{S}\right)_{1}^{*}, M_{I_{1}}^{*}, 0\right)
$$

where only strain 1 survives, and

$$
\mathcal{E}_{2}=\left(\left(H_{S}\right)_{j}^{*}, 0, H_{I_{j}}^{*}, 0, H_{S_{j 1}}^{*}, 0,0,\left(M_{S}\right)_{j}^{*}, 0, M_{I_{j}}^{*}\right)
$$

where only strain $j$ survives.

iii. Endemic Equilibrium (EE) given as

$$
\mathcal{E}_{3}=\left(H_{S}^{*}, H_{I_{1}}^{*}, H_{I_{j}}^{*}, H_{S_{1 j}}^{*}, H_{S_{j 1}}^{*}, H_{I_{1 j}}^{*}, H_{I_{j 1}}^{*}, M_{S}^{*}, M_{I_{1}}^{*}, M_{I_{j}}^{*}\right) .
$$

In this case, virus serotypes 1 and $j$ coexist.

\subsection{Global Stability of the Disease-Free Equilibrium}

Consider the DFE given by Equation (7). Adopting the next generation matrix method outlined in van den Driessche and Watmough [24], the basic reproduction number associated with Model (5) is given as

$$
\mathcal{R}_{0}^{2}=\max \left\{\mathcal{R}_{01}, \mathcal{R}_{0 j}\right\}
$$

with

$$
\mathcal{R}_{0 i}=\frac{B^{2} \beta_{H M_{i}} \beta_{M H_{i}} \mu_{M} M_{N}}{H_{N}\left(\mu_{M}+u_{2}\right)^{2}\left(\theta_{H_{i}}+\mu_{H}\right)}, \quad i=1, j,
$$

where $u_{2}$ is a constant parameter representing insecticide (adulticide) control measure, and $\mathcal{R}_{01}$ and $\mathcal{R}_{0 j}$ are the basic reproduction numbers related to virus serotypes 1 and $j$, respectively.

Now, we present Theorem 2 below to discuss the dynamical behaviour of Model (5) as its solutions tend to the disease free.

Theorem 2 If $\sigma_{1}, \sigma_{2} \leq 1$ and both $\mathcal{R}_{01}, \mathcal{R}_{0 j} \leq 1$, then the DFE $\mathcal{E}_{0}$ is Globally Asymptotically Stable $(G A S)$ in the region $\Gamma$ defined by Equation (6). 
Proof Consider the Lyapunov function of the form

$$
\begin{aligned}
\mathcal{W}= & \left(\mu_{M}+u_{2}\right) H_{I_{1}}+\left(\mu_{M}+u_{2}\right) H_{I_{j}}+\frac{\mathcal{R}_{0 j}^{2}\left(\mu_{M}+u_{2}\right)\left(\theta_{H_{j}}+\mu_{H}\right)}{\left(\theta_{H_{j}}+\mu_{H}+\mu_{D}\right)} H_{I_{1 j}} \\
& +\frac{\mathcal{R}_{01}^{2}\left(\mu_{M}+u_{2}\right)\left(\theta_{H_{1}}+\mu_{H}\right)}{\left(\theta_{H_{1}}+\mu_{H}+\mu_{D}\right)} H_{I_{j 1}}+B \beta_{M H_{1}} M_{I_{1}}+B \beta_{M H_{j}} M_{I_{j}} .
\end{aligned}
$$

The time derivative of $\mathcal{W}$ in Equation (13) along the solutions of Model (5), after some algebraic simplifications, is obtained as

$$
\begin{aligned}
\frac{d \mathcal{W}}{d t}= & -C-D+\left[B^{2} \beta_{M H_{1}} \beta_{H M_{1}} \frac{M_{S}}{H_{N}}-\left(\mu_{M}+u_{2}\right)\left(\theta_{H_{1}}+\mu_{H}\right)\right] H_{I_{1}} \\
& +\left[B^{2} \beta_{M H_{j}} \beta_{H M_{j}} \frac{M_{S}}{H_{N}}-\left(\mu_{M}+u_{2}\right)\left(\theta_{H_{j}}+\mu_{H}\right)\right] H_{I_{j}} \\
& +\left[B^{2} \beta_{M H_{1}} \beta_{H M_{1}} \frac{M_{S}}{H_{N}}-\mathcal{R}_{01}^{2}\left(\mu_{M}+u_{2}\right)\left(\theta_{H_{1}}+\mu_{H}\right)\right] H_{I_{j 1}} \\
& +\left[B^{2} \beta_{M H_{j}} \beta_{H M_{j}} \frac{M_{S}}{H_{N}}-\mathcal{R}_{0 j}^{2}\left(\mu_{M}+u_{2}\right)\left(\theta_{H_{j}}+\mu_{H}\right)\right] H_{I_{1 j}},
\end{aligned}
$$

with

$$
\begin{aligned}
& C=B \beta_{M H_{1}}\left(\mu_{M}+u_{2}\right) M_{I_{1}}\left[1-\frac{H_{S}}{H_{N}}-\frac{\sigma_{1}\left(\theta_{H_{1}}+\mu_{H}\right)}{\left(\theta_{H_{1}}+\mu_{H}+\mu_{D}\right)} \frac{H_{S_{j 1}}}{H_{N}}\right], \\
& D=B \beta_{M H_{j}}\left(\mu_{M}+u_{2}\right) M_{I_{j}}\left[1-\frac{H_{S}}{H_{N}}-\frac{\sigma_{j}\left(\theta_{H_{j}}+\mu_{H}\right) \mathcal{R}_{0 j}^{2}}{\left(\theta_{H_{j}}+\mu_{H}+\mu_{D}\right)} \frac{H_{S_{1 j}}}{H_{N}}\right] .
\end{aligned}
$$

At $M_{S} \leq M_{S}^{*}=\frac{\mu_{M} M_{N}}{\left(\mu_{M}+u_{2}\right)}$, it is clear that the last two terms in Equation (14) vanish. Also, note that $\frac{H_{S}}{H_{N}}+\frac{H_{S_{j 1}}}{H_{N}} \leq 1$ and $\frac{H_{S}}{H_{N}}+\frac{H_{S_{1 j}}}{H_{N}} \leq 1$. Hence, $\left[1-\frac{H_{S}}{H_{N}}-\frac{\sigma_{1}\left(\theta_{H_{1}}+\mu_{H}\right) \mathcal{R}_{01}^{2}}{\left(\theta_{H_{1}}+\mu_{H}+\mu_{D}\right)} \frac{H_{S_{j 1}}}{H_{N}}\right] \geq 0$ and $\left[1-\frac{H_{S}}{H_{N}}-\frac{\sigma_{j}\left(\theta_{H_{j}}+\mu_{H}\right) \mathcal{R}_{0 j}^{2}}{\left(\theta_{H_{j}}+\mu_{H}+\mu_{D}\right)} \frac{H_{S_{1 j}}}{H_{N}}\right] \geq 0$ if both $\sigma_{1}, \sigma_{j} \leq 1$. This implies that $C, D \geq 0$ in Equation (14). Consequently, $\frac{d \mathcal{W}}{d t} \leq 0$ if $\sigma_{1}, \sigma_{j} \leq 1$ and $E \leq 0$, where

$$
E=\left[\frac{B^{2} \beta_{M H_{i}} \beta_{H M_{i}} \mu_{M} M_{N}}{H_{N}\left(\mu_{M}+u_{2}\right)}-\left(\mu_{M}+u_{2}\right)\left(\theta_{H_{i}}+\mu_{H}\right)\right], \quad i=1, j .
$$

It follows from Equation (15) that

$$
E=\left(\mu_{M}+u_{2}\right)\left(\theta_{H_{i}}+\mu_{H}\right)\left(\mathcal{R}_{0 i}^{2}-1\right), \quad i=1, j .
$$

Therefore, $\frac{d \mathcal{W}}{d t} \leq 0$ if $\sigma_{i} \leq 1$ and $\mathcal{R}_{0 i}<1$ for $i=1, j$. In addition, $\frac{d \mathcal{W}}{d t}=0$ if and only if $H_{I_{i}}=0, M_{I_{i}}=0$ and $\mathcal{R}_{0 i}=1$ for $i=1, j$. This implies that the largest invariant set in $\left\{\left(H_{S}, H_{I_{1}}, H_{I_{j}}, H_{S_{1 j}}, H_{S_{j 1}}, H_{I_{1 j}}, H_{I_{j 1}}, M_{S}, M_{I_{1}}, M_{I_{j}}\right) \in \Gamma \frac{d \mathcal{W}}{d t}=0\right\}$ is the singleton set $\left\{\mathcal{E}_{0}\right\}$. Therefore, by LaSalle's invariance principle [25], the DFE $\mathcal{E}_{0}$ is GAS if $\sigma_{i} \leq 1$ and $\mathcal{R}_{0 i} \leq 1$, $i=1, j$. 


\subsection{Global Stability of the Boundary Equilibrium Points}

We consider the case when only a single strain of dengue virus survives in the interacting populations. Then, Model (5) decouples to the following Subsystem (17) for serotype $i$ (where $i=1, j)$ :

$$
\begin{aligned}
\frac{d\left(H_{S}\right)_{i}}{d t} & =\mu_{H} H_{N}-B \beta_{M H_{i}} \frac{M_{I_{i}}}{H_{N}}\left(H_{S}\right)_{i}-\mu_{H}\left(H_{S}\right)_{i}, \\
\frac{d H_{I_{i}}}{d t} & =B \beta_{M H_{i}} \frac{M_{I_{i}}}{H_{N}}\left(H_{S}\right)_{i}-\theta_{H_{i}} H_{I_{i}}-\mu_{H} H_{I_{i}}, \\
\frac{d H_{S_{i k}}}{d t} & =\theta_{H_{i}} H_{I_{i}}-\mu_{H} H_{S_{i k}}, \\
\frac{d\left(M_{S}\right)_{i}}{d t} & =\mu_{M} M_{N}-B \beta_{H M_{i}} \frac{H_{I_{i}}}{H_{N}}\left(M_{S}\right)_{i}-\mu_{M}\left(M_{S}\right)_{i}-u_{2}\left(M_{S}\right)_{i}, \\
\frac{d M_{I_{i}}}{d t} & =B \beta_{H M_{i}} \frac{H_{I_{i}}}{H_{N}}\left(M_{S}\right)_{i}-\mu_{M} M_{I_{i}}-u_{2} M_{I_{i}}, \quad \text { for } i, k=1, j, \quad i \neq k .
\end{aligned}
$$

Next, we obtain the EE points of Subsystem (17) for $i=1, j$ and discuss their global asymptotic stability. Let $\mathcal{E}_{4}$ and $\mathcal{E}_{5}$ be the EE points of Subsystem (17) for $i=1, j$, respectively. According to the theory of asymptotically autonomous system [26], if we could show that $\mathcal{E}_{4}$ and $\mathcal{E}_{5}$ are GAS on the sets

$$
\Gamma_{1}=\left\{\left(H_{S}, H_{I_{1}}, H_{S_{1 j}}, M_{S}, M_{I_{1}}\right) \in \mathbb{R}_{+}^{5} \mid H_{S}+H_{I_{1}}+H_{S_{1 j}}=H_{N} \text { and } M_{S}+M_{I_{1}}=M_{N}\right\}
$$

and

$$
\Gamma_{2}=\left\{\left(H_{S}, H_{I_{j}}, H_{S_{j 1}}, M_{S}, M_{I_{j}}\right) \in \mathbb{R}_{+}^{5} \mid H_{S}+H_{I_{j}}+H_{S_{j 1}}=H_{N} \text { and } M_{S}+M_{I_{j}}=M_{N}\right\}
$$

respectively, then it would imply that the BE points $\mathcal{E}_{1}$ and $\mathcal{E}_{2}$ are GAS for Model (5) on the set $\Gamma$ given in Equation (6). This is because the unique EE points $\mathcal{E}_{4}$ and $\mathcal{E}_{5}$, respectively, are equivalent to the $\mathrm{BE}$ points $\mathcal{E}_{1}$ and $\mathcal{E}_{2}$ of Model (5). We find $\mathcal{E}_{4}$ and $\mathcal{E}_{5}$ as given by

$$
\mathcal{E}_{4}=\left(\left(H_{S}\right)_{1}^{*}, H_{I_{1}}^{*}, H_{S_{1 j}}^{*},\left(M_{S}\right)_{1}^{*}, M_{I_{1}}^{*}\right)
$$

and

$$
\mathcal{E}_{5}=\left(\left(H_{S}\right)_{j}^{*}, H_{I_{j}}^{*}, H_{S_{j 1}}^{*},\left(M_{S}\right)_{j}^{*}, M_{I_{j}}^{*}\right)
$$

where,

$$
\begin{aligned}
H_{I_{i}}^{*} & =\frac{\left(\mu_{M}+u_{2}\right)^{2} \mu_{H} H_{N}\left(\mathcal{R}_{0 i}^{2}-1\right)}{\left[\left(\mu_{M}+u_{2}\right) \mu_{H} H_{N}+B \beta_{M H_{i}} \mu_{M} M_{N}\right] B \beta_{H M_{i}}} H_{N}, \\
H_{S_{i k}}^{*} & =\frac{\theta_{H_{i}}}{\mu_{H}} H_{I_{i}}^{*}, \quad\left(H_{S}\right)_{i}^{*}=H_{N}-\frac{\left(\theta_{H_{i}}+\mu_{H}\right)}{\mu_{H}} H_{I_{i}}^{*}, \\
\left(M_{S}\right)_{i}^{*} & =\frac{\mu_{M} M_{N}}{\left[\mu_{M}+u_{2}+B \beta_{H M_{i}} \frac{H_{I_{N}}^{*}}{H_{N}}\right]}, M_{I_{i}}^{*}=\frac{B \beta_{H M_{i}} \mu_{M} M_{N} \frac{H_{I_{i}}^{*}}{\left(\mu_{M}+u_{2}\right)\left[\mu_{M}+u_{2}+B \beta_{H M_{i}} \frac{H_{I_{i}}^{*}}{H_{N}}\right]},}{}
\end{aligned}
$$


for $i, k=1, j, \quad i \neq k$.

Hence, the mathematical expressions for the BE points $\mathcal{E}_{1}$ and $\mathcal{E}_{2}$ expressed by Equations (8) and (9), respectively, are immediately obtained as

$$
\begin{aligned}
& \mathcal{E}_{1}=\left(\left(H_{S}\right)_{1}^{*}, H_{I_{1}}^{*}, 0, H_{S_{1 j}}^{*}, 0,0,0,\left(M_{S}\right)_{1}^{*}, M_{I_{1}}^{*}, 0\right), \\
& \mathcal{E}_{2}=\left(\left(H_{S}\right)_{j}^{*}, 0, H_{I_{j}}^{*}, 0, H_{S_{j 1}}^{*}, 0,0,\left(M_{S}\right)_{j}^{*}, 0, M_{I_{j}}^{*}\right),
\end{aligned}
$$

where the components of $\mathcal{E}_{1}$ and $\mathcal{E}_{2}$ are as obtained in Equation (22).

Now, the global asymptotic stability of the EE $\mathcal{E}_{4}$ of Subsystem (17) for $i=1$, given by Equation (20), is presented by the following Theorem 3.

Theorem 3 Whenever $\mathcal{R}_{01}>1$, then the EE $\mathcal{E}_{4}$ of Subsystem (17) (for $i=1$ ) is GAS in the region $\Gamma_{1}$.

Proof Consider the Goh-Volterra type Lyapunov function [27] given as

$$
\begin{aligned}
\mathcal{V}= & d_{1}\left(H_{S}-H_{S}^{*}-H_{S}^{*} \ln \frac{H_{S}}{H_{S}^{*}}\right)+d_{2}\left(H_{I_{1}}-H_{I_{1}}^{*}-H_{I_{1}}^{*} \ln \frac{H_{I_{1}}}{H_{I_{1}}^{*}}\right) \\
& +d_{3}\left(M_{S}-M_{S}^{*}-M_{S}^{*} \ln \frac{M_{S}}{M_{S}^{*}}\right)+d_{4}\left(M_{I_{1}}-M_{I_{1}}^{*}-M_{I_{1}}^{*} \ln \frac{M_{I_{1}}}{M_{I_{1}}^{*}}\right) .
\end{aligned}
$$

With suitably determined coefficients, $d_{1}=d_{2}=\frac{B \beta_{H M_{1}} M_{S}^{*}}{H_{N}}$ and $d_{3}=d_{4}=\frac{B \beta_{M H_{1}} M_{I_{1}}^{*} H_{S}^{*}}{H_{N} H_{I_{1}}^{*}}$, the time derivative of $\mathcal{V}$ in Equation (23) along the solutions of Subsystem (17) yields

$$
\begin{aligned}
\frac{d \mathcal{V}}{d t}= & \frac{B \beta_{H M_{1}} M_{S}^{*}}{H_{N}}\left(\mu_{H} H_{S}^{*}\left(2-\frac{H_{S}}{H_{S}^{*}}-\frac{H_{S}^{*}}{H_{S}}\right)+\frac{B \beta_{M H_{1}} M_{I_{1}}^{*} H_{S}^{*}}{H_{N}}-\frac{B \beta_{M H_{1}} M_{I_{1}}^{*} H_{S}^{*}}{H_{N}} \frac{H_{S}^{*}}{H_{S}}\right) \\
& +\frac{B \beta_{H M_{1}} M_{S}^{*}}{H_{N}}\left\{-\frac{B \beta_{M H_{1}} H_{I_{1}}^{*}}{H_{N}} \frac{M_{I_{1}} H_{S}}{H_{I_{1}}}+\left(\theta_{H_{1}}+\mu_{H}\right) H_{I_{1}}^{*}\right\} \\
& +\frac{B \beta_{M H_{1}} M_{I_{1}}^{*} H_{S}^{*}}{H_{N} H_{I_{1}}^{*}}\left\{\left(\mu_{M}+u_{2}\right) M_{S}^{*}\left(2-\frac{M_{S}}{M_{S}^{*}}-\frac{M_{S}^{*}}{M_{S}}\right)+\frac{B \beta_{H M_{1}} H_{I_{1}}^{*} M_{S}^{*}}{H_{N}}\right. \\
& \left.-\frac{B \beta_{H M_{1}} H_{I_{1}}^{*} M_{S}^{*}}{H_{N}} \frac{M_{S}^{*}}{M_{S}}\right\}+\frac{B \beta_{M H_{1}} M_{I_{1}}^{*} H_{S}^{*}}{H_{N} H_{I_{1}}^{*}}\left\{\left(\mu_{M}+u_{2}\right) M_{I_{1}}^{*}-\frac{B \beta_{H M_{1}} M_{I_{1}}^{*} H_{I_{1}} M_{S}}{H_{N} M_{I_{1}}}\right\} .
\end{aligned}
$$

At steady state, the following relations hold from Subsystem (17):

$$
\begin{aligned}
\mu_{H} H_{N} & =B \beta_{M H_{1}} \frac{M_{I_{1}}^{*}}{H_{N}} H_{S}^{*}+\mu_{H} H_{S}^{*}, \theta_{H_{1}}+\mu_{H}=\frac{B \beta_{M H_{1}} M_{I_{1}}^{*} H_{S}^{*}}{H_{N} H_{I_{1}}^{*}}, \\
\mu_{M} M_{N} & =B \beta_{H M_{1}} \frac{H_{I_{1}}^{*}}{H_{N}} M_{S}^{*}+\left(\mu_{M}+u_{2}\right) M_{S}^{*}, \mu_{M}+u_{2}=\frac{B \beta_{H M_{1}} H_{I_{1}}^{*} M_{S}^{*}}{H_{N} M_{I_{1}^{*}}} .
\end{aligned}
$$

Using Equation (25) in Equation (24) and simplifying lead to

$$
\begin{aligned}
\frac{d \mathcal{V}}{d t}= & \frac{\mu_{H} B \beta_{H M_{1}} M_{S}^{*} H_{S}^{*}}{H_{N}}\left(2-\frac{H_{S}}{H_{S}^{*}}-\frac{H_{S}^{*}}{H_{S}}\right)+\frac{B^{2} \beta_{M H_{1}} \beta_{H M_{1}} H_{S}^{*} M_{S}^{* 2}}{H_{N}^{2}}\left(2-\frac{M_{S}}{M_{S}^{*}}-\frac{M_{S}^{*}}{M_{S}}\right) \\
& +\frac{B^{2} \beta_{M H_{1}} \beta_{H M_{1}} M_{I_{1}}^{*} M_{S}^{*} H_{S}^{*}}{H_{N}^{2}}\left(4-\frac{H_{S}^{*}}{H_{S}}-\frac{M_{S}^{*}}{M_{S}}-\frac{H_{I_{1}}^{*} M_{I_{1}} H_{S}}{H_{I_{1}} M_{I_{1}}^{*} H_{S}^{*}}-\frac{M_{I_{1}}^{*} M_{S} H_{I_{1}}}{M_{I_{1}} M_{S}^{*} H_{I_{1}}^{*}}\right) .
\end{aligned}
$$


Hence, since arithmetic mean $\geq$ geometric mean, we have

$$
\left(2-\frac{H_{S}}{H_{S}^{*}}-\frac{H_{S}^{*}}{H_{S}}\right) \leq 0,\left(2-\frac{M_{S}}{M_{S}^{*}}-\frac{M_{S}^{*}}{M_{S}}\right) \leq 0,\left(4-\frac{H_{S}^{*}}{H_{S}}-\frac{M_{S}^{*}}{M_{S}}-\frac{H_{I_{1}}^{*} M_{I_{1}} H_{S}}{H_{I_{1}} M_{I_{1}}^{*} H_{S}^{*}}-\frac{M_{I_{1}}^{*} M_{S} H_{I_{1}}}{M_{I_{1}} M_{S}^{*} H_{I_{1}}^{*}}\right) \leq 0 .
$$

Therefore, $\frac{d \mathcal{V}}{d t} \leq 0$ since all the model parameters are positive. Furthermore, $\frac{d \mathcal{V}}{d t}=0$ if and only if $H_{S}=H_{S}^{*}, H_{I_{1}}=H_{I_{1}}^{*}, M_{S}=M_{S}^{*}, M_{I_{1}}=M_{I_{1}}^{*}$. It then follows that $H_{S_{1 j}} \rightarrow H_{S_{1 j}}^{*}$ as $t \rightarrow \infty$, and by LaSalle's invariance principle, the EE $\mathcal{E}_{4}$ is GAS whenever $\mathcal{R}_{01}>1$. This completes the proof.

Consequently, the global asymptotic stability of the $\mathrm{BE} \mathcal{E}_{1}$ is summarized in the following result.

Theorem 4 The BE $\mathcal{E}_{1}$ of Model (5), presented by Equation (8), is GAS in the region $\Gamma$ if $\mathcal{R}_{01}>1$, and unstable otherwise.

Proof The proof of this theorem is similar to that of Theorem 3.

Using the results of Theorems 3 and 4, the global asymptotic stability of the EE and BE points $\mathcal{E}_{2}$ and $\mathcal{E}_{5}$ are presented in the next two theorems, respectively.

Theorem 5 The EE $\mathcal{E}_{5}$ of Subsystem (17) (for $i=j$ ) is $G A S$ in the region $\Gamma_{2}$, given by Equation (19), if $\mathcal{R}_{0 j}>1$. Otherwise, it is unstable.

Theorem 6 The $B E \mathcal{E}_{2}$ associated with Model (5), defined by Equation (9), is GAS in the region $\Gamma$ whenever $\mathcal{R}_{0 j}>1$, and unstable otherwise.

Proof The proof of Theorems 5 and 6 can be established by using the same approach adopted in proving Theorems 3 and 4.

Theorems 3 and 4 epidemiologically implicate that when virus serotype 1 is predominant, dengue disease invades the interacting human and mosquito populations whenever $\mathcal{R}_{01}>1$ regardless of the value of initial sizes of the infected subgroups in the populations. Similarly, Theorems 5 and 6 suggest that dengue disease with virus serotype $j$ persists in the population when $\mathcal{R}_{0 j}>1$ irrespective of the initial data values of the infected classes in the populations.

In the next section, we formulate OC problem for the dynamics of dengue spread.

\section{Formulation of Optimal Control Problem}

In this section, we present the OC problem formulation for the dynamics of dengue transmission in order to derive the optimal levels of the time-dependent controls $u_{1}(t)$ and $u_{2}(t)$ that minimize the number of infected individuals, total mosquito population and costs of control implementation. Thus, our specific objective is to determine an OC strategy for prevention and control of dengue spread using OC theory.

First, we standardized Model (3) by using the following scales:

$$
\begin{aligned}
& x_{1}=\frac{H_{S}}{H_{N}}, \quad x_{2}=\frac{H_{I_{1}}}{H_{N}}, \quad x_{3}=\frac{H_{I_{j}}}{H_{N}}, \quad x_{4}=\frac{H_{S_{1 j}}}{H_{N}}, \quad x_{5}=\frac{H_{S_{j 1}}}{H_{N}}, \quad x_{6}=\frac{H_{I_{1 j}}}{H_{N}}, \\
& x_{7}=\frac{H_{I_{j 1}}}{H_{N}}, \quad x_{8}=\frac{H_{R}}{H_{N}}, \quad x_{9}=\frac{M_{S}}{m H_{N}}, \quad x_{10}=\frac{M_{I_{1}}}{m H_{N}}, \quad x_{11}=\frac{M_{I_{j}}}{m H_{N}} .
\end{aligned}
$$


So, Model (3) becomes

$$
\begin{aligned}
& \frac{d x_{1}(t)}{d t}=\mu_{H}-\left(m B\left(\beta_{M H_{1}} x_{10}(t)+\beta_{M H_{j}} x_{11}(t)\right)+\mu_{H}\right) x_{1}(t), \\
& \frac{d x_{2}(t)}{d t}=m B \beta_{M H_{1}} x_{1}(t) x_{10}(t)-\left(\theta_{H_{1}}+\mu_{H}\right) x_{2}(t), \\
& \frac{d x_{3}(t)}{d t}=m B \beta_{M H_{j}} x_{1}(t) x_{11}(t)-\left(\theta_{H_{j}}+\mu_{H}\right) x_{3}(t), \\
& \frac{d x_{4}(t)}{d t}=\theta_{H_{1}} x_{2}(t)-\left(\sigma_{j} m B \beta_{M H_{j}} x_{11}(t)+\mu_{H}+u_{1}(t)\right) x_{4}(t), \\
& \frac{d x_{5}(t)}{d t}=\theta_{H_{j}} x_{3}(t)-\left(\sigma_{1} m B \beta_{M H_{1}} x_{10}(t)+\mu_{H}+u_{1}(t)\right) x_{5}(t), \\
& \frac{d x_{6}(t)}{d t}=\sigma_{j} m B \beta_{M H_{j}} x_{4}(t) x_{11}(t)-\left(\theta_{H_{j}}+\mu_{H}+\mu_{D}\right) x_{6}(t), \\
& \frac{d x_{7}(t)}{d t}=\sigma_{1} m B \beta_{M H_{1}} x_{5}(t) x_{10}(t)-\left(\theta_{H_{1}}+\mu_{H}+\mu_{D}\right) x_{7}(t), \\
& \frac{d x_{8}(t)}{d t}=\theta_{H_{j}} x_{6}(t)+\theta_{H_{1}} x_{7}(t)+u_{1}(t) x_{4}(t)+u_{1}(t) x_{5}(t)-\mu_{H} x_{8}(t), \\
& \frac{d x_{9}(t)}{d t}=\mu_{M}-\left(B \beta_{H M_{1}}\left(x_{2}(t)+x_{7}(t)\right)+B \beta_{H M_{j}}\left(x_{3}(t)+x_{6}(t)\right)+\mu_{M}+u_{2}(t)\right) x_{9}(t), \\
& \frac{d x_{10}(t)}{d t}=B \beta_{H M_{1}}\left(x_{2}(t)+x_{7}(t)\right) x_{9}(t)-\left(\mu_{M}+u_{2}(t)\right) x_{10}(t), \\
& \frac{d x_{11}(t)}{d t}=B \beta_{H M_{j}}\left(x_{3}(t)+x_{6}(t)\right) x_{9}(t)-\left(\mu_{M}+u_{2}(t)\right) x_{11}(t),
\end{aligned}
$$

together with the ICs:

$$
\begin{aligned}
& x_{1}(0)=x_{01}, \quad x_{2}(0)=x_{02}, \quad x_{3}(0)=x_{03}, \quad x_{4}(0)=x_{04}, \quad x_{5}(0)=x_{05}, \quad x_{6}(0)=x_{06}, \\
& x_{7}(0)=x_{07}, \quad x_{8}(0)=x_{08}, \quad x_{9}(0)=x_{09}, \quad x_{10}(0)=x_{010}, \quad x_{11}(0)=x_{011} \text {. }
\end{aligned}
$$

Here,

$$
x_{1}(t)+x_{2}(t)+x_{3}(t)+x_{4}(t)+x_{5}(t)+x_{6}(t)+x_{7}(t)+x_{8}(t)=1
$$

and

$$
x_{9}(t)+x_{10}(t)+x_{11}(t)=1 .
$$

The normalized system, Equation (28) along with ICs (29), allows us to deal with population proportions instead of the actual population sizes without any loss of generality. As from now on, we let $y(t)=y$, where $y(t) \in\left\{x_{1}(t), x_{2}(t), \cdots, x_{11}(t), u_{1}(t), u_{2}(t)\right\}$.

In the formulation of $\mathrm{OC}$ problem, the cost functional $(\mathrm{CF})$ is constructed such that it is quadratic in the control terms. This is in line with other studies [20-22]. Hence, we present our OC problem as follows:

$$
\min J\left(u_{1}, u_{2}\right)=\int_{0}^{t_{f}} \mathcal{L}\left(t, x_{2}, x_{3}, x_{6}, x_{7}, x_{9}, x_{10}, x_{11}, u_{1}, u_{2}\right) d t
$$


subject to System (28) together with the state ICs, Equation (29). The Lagrangian $\mathcal{L}$, in Equation (30), is given by

$$
\begin{aligned}
\mathcal{L}\left(t, x_{2}, x_{3}, x_{6}, x_{7}, x_{9}, x_{10}, x_{11}, u_{1}, u_{2}\right)= & w_{1}\left(x_{2}+x_{7}\right)+w_{2}\left(x_{3}+x_{6}\right)+w_{3} x_{9}+w_{4} x_{10} \\
& +w_{5} x_{11}+\frac{1}{2} w_{6} u_{1}^{2}+\frac{1}{2} w_{7} u_{2}^{2}
\end{aligned}
$$

where $u_{1}$ and $u_{2}$ represent the rates of vaccinating seropositive individuals and adulticiding at time $t$, respectively, $t_{f}$ is the final time for administering the control interventions, and the admissible control set $\mathbf{u}$, with $u_{i \max }=0.9$, is defined as

$$
\mathbf{u}=\left\{\left(u_{1}, u_{2}\right) \mid u_{i} \text { is Lebesgue measurable, } 0 \leq u_{i} \leq u_{i \max } \leq 1, i=1,2\right\}
$$

We chose $u_{i \max }=0.9$ because it is more realistic to admit the possibility of vaccinating $90 \%$ rather than $100 \%$ of seropositive individuals. Also, 90\% level of administering adulticide is practical.

The CF specification involves the number of infected individuals, total mosquito population and cost related to the application of controls $u_{1}$ and $u_{2}$. In the Lagrangian $\mathcal{L}$ in Equation (31), the coefficients $w_{i}(i=1,2, \cdots, 5)$ are the weight constants associated with proportions of primary and secondary infected individuals with virus serotype 1, primary and secondary infected individuals with virus serotype $j$, susceptible mosquitoes, and infected mosquitoes with virus serotype 1 and serotype $j$, respectively. They help to balance each term of the integrand so that none of them dominates. The quantities $w_{6}$ and $w_{7}$ are the weight constants for vaccination and adulticide, respectively. The costs associated with controls $u_{1}$ and $u_{2}$ are respectively represented by the terms $\frac{1}{2} w_{6} u_{1}^{2}$ and $\frac{1}{2} w_{7} u_{2}^{2}$. However, $w_{i}(i=1,2, \cdots, 5)$ are the measures of the importance of reducing the associated states on the disease transmission, while $w_{6}$ and $w_{7}$ are the relative measures of the costs or efforts required to implement the respective controls. In Subsections 4.1 and 4.2, we discuss the existence and characterization of OCs.

\subsection{Existence of Optimal Controls}

In this subsection, the sufficient condition for the existence of a solution to the OC problem is considered.

Theorem 7 There exists an $O C$ set $\mathbf{u}^{*}=\left(u_{1}^{*}, u_{2}^{*}\right)$ with a corresponding states solution $\left(x_{1}^{*}, x_{2}^{*}, x_{3}^{*}, x_{4}^{*}, x_{5}^{*}, x_{6}^{*}, x_{7}^{*}, x_{8}^{*}, x_{9}^{*}, x_{10}^{*}, x_{11}^{*}\right)$ to System (28) such that

$$
\min _{\left(u_{1}, u_{2}\right) \in \mathbf{u}} J\left(u_{1}, u_{2}\right)=J\left(u_{1}^{*}, u_{2}^{*}\right) .
$$

Proof Similar to the proof of Theorem 4.1 in Khan et al. [28].

\subsection{Characterization of Optimal Controls}

Here, the OCs $\left(u_{1}^{*}, u_{2}^{*}\right)$ that give the optimal levels for the control variables and the corresponding optimal states $\left(x_{1}^{*}, x_{2}^{*}, x_{3}^{*}, x_{4}^{*}, x_{5}^{*}, x_{6}^{*}, x_{7}^{*}, x_{8}^{*}, x_{9}^{*}, x_{10}^{*}, x_{11}^{*}\right)$ are characterized. The necessary conditions to be satisfied by the OCs are derived using PMP [29]. This principle 
converts the CF in Equation (30) and the constraints, System (28), into a problem of pointwise minimization of a Hamiltonian $\mathcal{H}$ with respect to the controls $u_{1}$ and $u_{2}$, where $\mathcal{H}$ is defined as

$$
\begin{aligned}
\mathcal{H}= & w_{1}\left(x_{2}+x_{7}\right)+w_{2}\left(x_{3}+x_{6}\right)+w_{3} x_{9}+w_{4} x_{10}+w_{5} x_{11}+\frac{1}{2} w_{6} u_{1}^{2}+\frac{1}{2} w_{7} u_{2}^{2} \\
& +\sum_{i=1}^{11} \lambda_{i}^{T} g_{i}\left(t, x_{1}, x_{2}, x_{3}, x_{4}, x_{5}, x_{6}, x_{7}, x_{8}, x_{9}, x_{10}, x_{11}\right) .
\end{aligned}
$$

In Equation (34), $g_{i}$ is the right-hand side of System $(28)$ and $\lambda_{i}$ (where $i=1,2, \ldots, 11$ ) are the adjoint (or costate) variables.

Theorem 8 (Necessary Conditions) Let $\left(u_{1}^{*}, u_{2}^{*}\right) \in \mathbf{u}$ be an OC pair with the corresponding optimal states $\left(x_{1}^{*}, x_{2}^{*}, x_{3}^{*}, x_{4}^{*}, x_{5}^{*}, x_{6}^{*}, x_{7}^{*}, x_{8}^{*}, x_{9}^{*}, x_{10}^{*}, x_{11}^{*}\right)$. Then, there exist costate variables $\lambda_{i}$ $(i=1,2, \ldots, 11)$ that satisfy

$$
\begin{aligned}
\frac{d \lambda_{1}}{d t} & =\lambda_{1} \mu_{H}+\left(\lambda_{1}-\lambda_{2}\right) m B \beta_{M H_{1}} x_{10}+\left(\lambda_{1}-\lambda_{3}\right) m B \beta_{M H_{j}} x_{11}, \\
\frac{d \lambda_{2}}{d t} & =\lambda_{2} \mu_{H}+\left(\lambda_{2}-\lambda_{4}\right) \theta_{H_{1}}+\left(\lambda_{9}-\lambda_{10}\right) B \beta_{H M_{1}} x_{9}-w_{1}, \\
\frac{d \lambda_{3}}{d t} & =\lambda_{3} \mu_{H}+\left(\lambda_{3}-\lambda_{5}\right) \theta_{H_{j}}+\left(\lambda_{9}-\lambda_{11}\right) B \beta_{H M_{j}} x_{9}-w_{2}, \\
\frac{d \lambda_{4}}{d t} & =\lambda_{4} \mu_{H}+\left(\lambda_{4}-\lambda_{6}\right) \sigma_{j} m B \beta_{M H_{j}} x_{11}+\left(\lambda_{4}-\lambda_{8}\right) u_{1}, \\
\frac{d \lambda_{5}}{d t} & =\lambda_{5} \mu_{H}+\left(\lambda_{5}-\lambda_{7}\right) \sigma_{1} m B \beta_{M H_{1}} x_{10}+\left(\lambda_{5}-\lambda_{8}\right) u_{1}, \\
\frac{d \lambda_{6}}{d t} & =\lambda_{6}\left(\mu_{H}+\mu_{D}\right)+\left(\lambda_{6}-\lambda_{8}\right) \theta_{H_{j}}+\left(\lambda_{9}-\lambda_{11}\right) B \beta_{H M_{j}} x_{9}-w_{2}, \\
\frac{d \lambda_{7}}{d t} & =\lambda_{7}\left(\mu_{H}+\mu_{D}\right)+\left(\lambda_{7}-\lambda_{8}\right) \theta_{H_{1}}+\left(\lambda_{9}-\lambda_{10}\right) B \beta_{H M_{1}} x_{9}-w_{1}, \\
\frac{d \lambda_{8}}{d t} & =\lambda_{8} \mu_{H}, \\
\frac{d \lambda_{9}}{d t} & =\lambda_{9}\left(\mu_{M}+u_{2}\right)+\left(\lambda_{9}-\lambda_{10}\right) B \beta_{H M_{1}}\left(x_{2}+x_{7}\right)+\left(\lambda_{9}-\lambda_{11}\right) B \beta_{H M_{j}}\left(x_{3}+x_{6}\right)-w_{3}, \\
\frac{d \lambda_{10}}{d t} & =\left(\lambda_{1}-\lambda_{2}\right) m B \beta_{M H_{1}} x_{1}+\left(\lambda_{5}-\lambda_{7}\right) \sigma_{1} m B \beta_{m H_{1}} x_{5}+\lambda_{10}\left(\mu_{M}+u_{2}\right)-w_{4}, \\
\frac{d \lambda_{11}}{d t} & =\left(\lambda_{1}-\lambda_{3}\right) m B \beta_{M H_{j}} x_{1}+\left(\lambda_{4}-\lambda_{6}\right) \sigma_{j} m B \beta_{M H_{j}} x_{4}-w_{5},
\end{aligned}
$$

and the transversality (or boundary) conditions:

$$
\lambda_{i}\left(t_{f}\right)=0,(i=1,2, \ldots, 11),
$$

with the OCs defined by

$$
\begin{aligned}
& u_{1}^{*}=\min \left\{\max \left\{0, \frac{\left(\lambda_{4}-\lambda_{8}\right) x_{4}^{*}+\left(\lambda_{5}-\lambda_{8}\right) x_{5}^{*}}{w_{6}}\right\}, 0.9\right\}, \\
& u_{2}^{*}=\min \left\{\max \left\{0, \frac{\lambda_{9} x_{9}^{*}+\lambda_{10} x_{10}^{*}+\lambda_{11} x_{11}^{*}}{w_{7}}\right\}, 0.9\right\} .
\end{aligned}
$$


Proof Using PMP [29], Equation (35) is obtained from

$$
-\frac{d \lambda_{i}}{d t}=\frac{\partial \mathcal{H}}{\partial x_{i}} \quad(i=1,2, \ldots, 11) .
$$

Also, it is clear from Equation (30) that the transversality conditions have the form (36), since all the states are free at the terminal time $t_{f}$. The Hamiltonian $\mathcal{H}$ is minimized with respect to the controls at $\mathbf{u}^{*}=\left(u_{1}^{*}, u_{2}^{*}\right)$ by solving

$$
\frac{\partial \mathcal{H}}{\partial u_{i}}=0 \quad \text { at } \quad u_{i}=u_{i}^{*}, \quad \text { for } i=1,2
$$

respectively, to obtain

$$
\begin{aligned}
& \frac{\partial \mathcal{H}}{\partial u_{1}}=w_{6} u_{1}-\left(\lambda_{4}-\lambda_{8}\right) x_{4}-\left(\lambda_{5}-\lambda_{8}\right) x_{5}=0, \text { at } u_{1}=u_{1}^{*}, \\
& \frac{\partial \mathcal{H}}{\partial u_{2}}=w_{7} u_{2}-\lambda_{9} x_{9}-\lambda_{10} x_{10}-\lambda_{11} x_{11}, \quad \text { at } u_{2}=u_{2}^{*} .
\end{aligned}
$$

Now, solving Equation (38) for $u_{i}^{*}(i=1,2)$ and imposing the bounds $0 \leq u_{i} \leq 0.9(i=1,2)$ on the results obtained yield the characterization of the OC in Equation (37).

Next, we find the $\mathrm{OC}$ and state by solving the optimality system which consists of System (28), initial conditions (29), the costate system (35), boundary conditions (36) and the characterized OCs in Equation (37) numerically. It is clear from Equation (38) that the second derivative of $\mathcal{H}$ with respect to $u_{1}$ and $u_{2}$ are positive. This indicates that both $u_{1}^{*}$ and $u_{2}^{*}$ are minimizers for the OC problem, Equation (30) subject to System (28).

\subsection{Numerical Simulations}

In this subsection, numerical simulation is carried out on the resulting optimality system in order to obtain the optimal strategy for the minimization of dengue disease spread and control costs. The optimality system is solved numerically in MATLAB with ode45 routine using a fourth-order Runge-Kutta scheme based on the forward-backward-sweep procedure. For details about this method, see Lenhart and Workman [30]. The initial values for the system of ODEs representing human population: $H_{S}(0)=109809, H_{I_{1}}(0)=10, H_{I_{j}}(0)=10, H_{S_{1 j}}(0)=2161$, $H_{S_{j 1}}(0)=0, H_{I_{1 j}}(0)=10, H_{I_{j 1}}(0)=0$ and $H_{R}(0)=0$ are taken from [4], while those related to mosquito population are taken as $M_{I_{1}}(0)=1000$ [21], $I_{M_{j}}(0)=1000$ and $M_{S}=670000$. With these values, we obtain the respective scaled initial values, Equation (29), for System (28) using Equation (27). There are two possibilities of dengue scenario when a second disease outbreak occur in Madeira Island, namely less aggressive and more aggressive cases [4], referred to as Scenarios 1 and 2, respectively. In Scenario $1, j=4$ and the values of $\frac{1}{\theta_{H_{j}}}, \beta_{M H_{j}}$ are both lower than those of $\frac{1}{\theta_{H_{1}}}, \beta_{M H_{1}}$. Also, $j=2 / 3$ and both the values of $\frac{1}{\theta_{H_{j}}}, \beta_{M H_{j}}$ are greater than those of $\frac{1}{\theta_{H_{1}}}, \beta_{M H_{1}}$ in Scenario 2. Hence, the model parameter values used for our simulations are as described in Table 1. Also, we take the state and control balancing weight values as $w_{1}=25$, $w_{2}=15, w_{3}=25, w_{4}=1, w_{5}=5, w_{6}=25$ and $w_{7}=25$ in both scenarios. For the control interventions, we implement the three strategies for each scenario. The results of our numerical implementation are presented and discussed in the next section. 


\section{Results and Discussion}

In the current section, we present and discuss the results of numerical implementation on the optimality system.

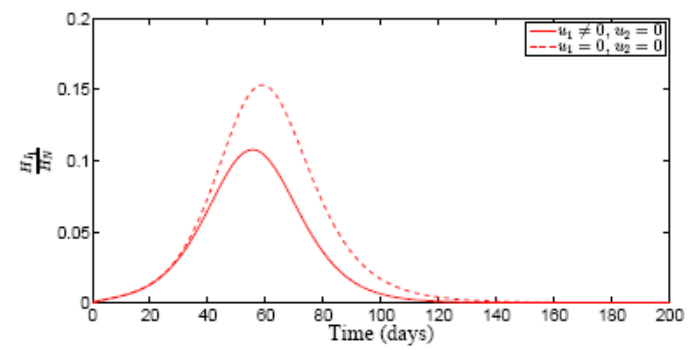

(a) Infected Humans by DEN-1

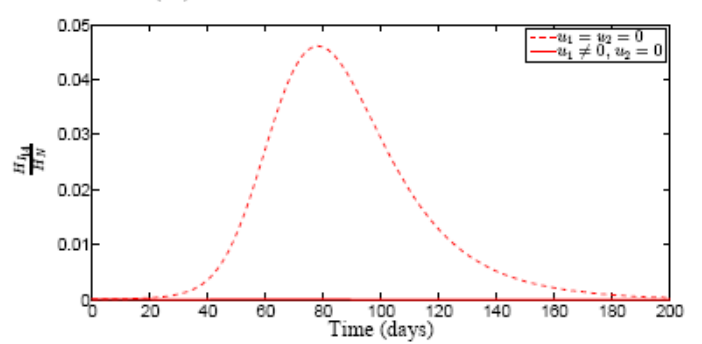

(c) Infected Humans by DEN-1, then by DEN-4

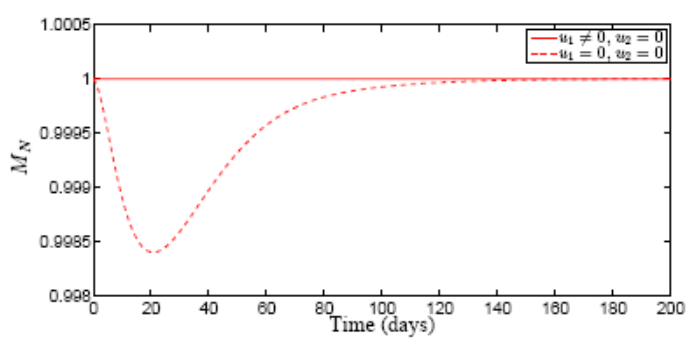

(e) Total Mosquito Population

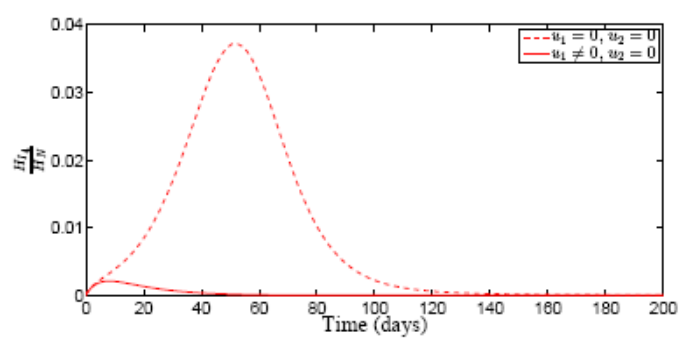

(b) Infected Humans by DEN-4

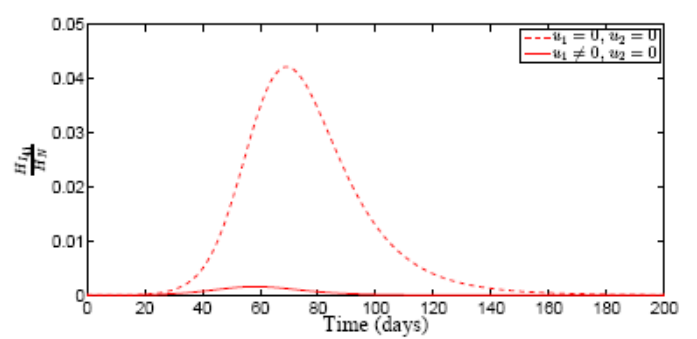

(d) Infected Humans by DEN-4, then by DEN-1

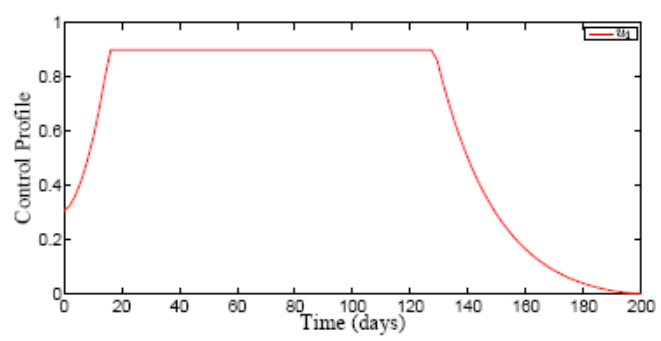

(f) Optimal Control Profile for $u_{1}$

Figure 1: Simulations of System (28) Displaying the Impact of $u_{1}$ (Dengvaxia Vaccine) on Dengue Spread with Coexistence of DEN-1 and DEN-4 for Scenario 1

Figures 1-3 present the results of our numerical simulations for Scenario 1. Figure 1 illustrates the impacts of control $u_{1}$ on the dynamics of primary and secondary infected subpopulations of human and total mosquito population. Unlike in the absence of control, the sizes of the primary and secondary infected humans with control decline more rapidly with higher number of secondary infected humans as seen in Figures 1a-1d. However, Figure 1eshows that control $u_{1}$ has less effect on mosquito as the population of mosquito with control remained constant over time. Also, it is observed from the control profile in Figure if that the OC $u_{1}$ is at the upper bound between the 15th and 128th days after which it drops to the lower bound at the final time. Our results agree with the results of the study on the optimal strategy for preventing and controlling a single strain dengue epidemic using vaccination by Rodrigues et al. [9], which revealed that the number of infected humans can be reduced close to zero by keeping vaccination control at the upper bound. 


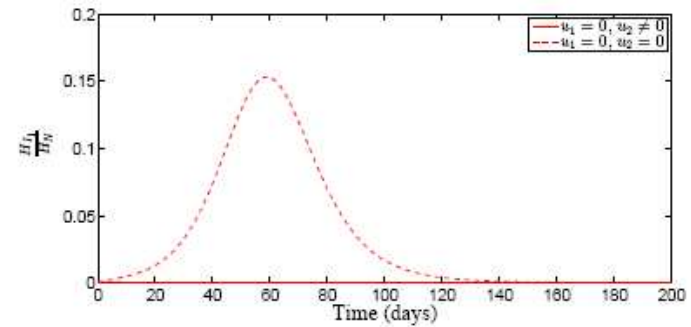

(a) Infected Humans by DEN-1

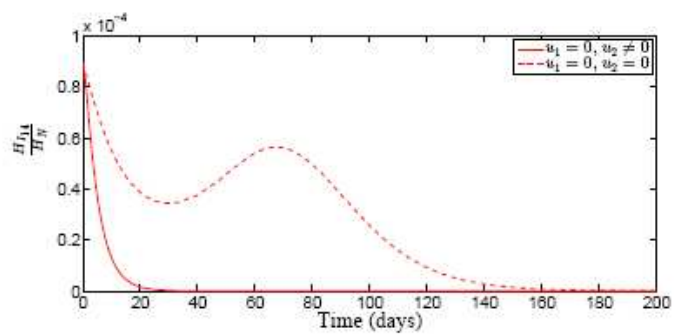

(c) Infected Humans by DEN-1, then by DEN-4

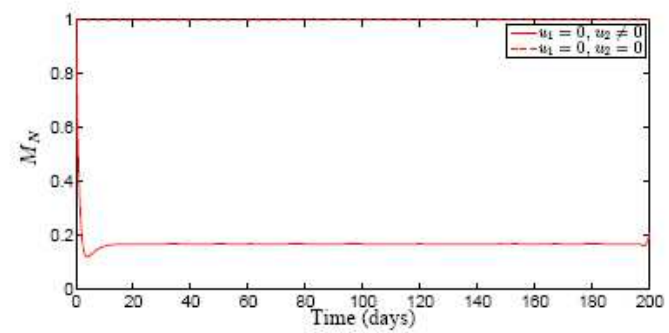

(e) Total Mosquito Population

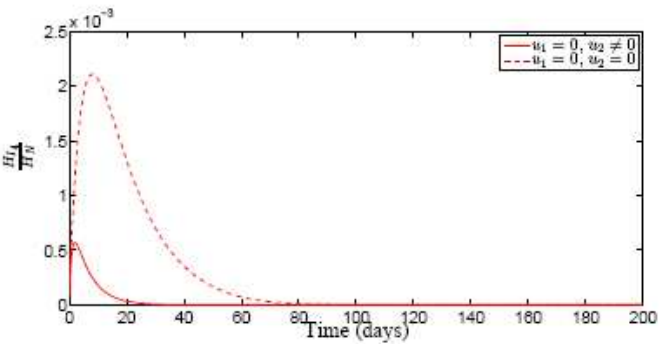

(b) Infected Humans by DEN-4

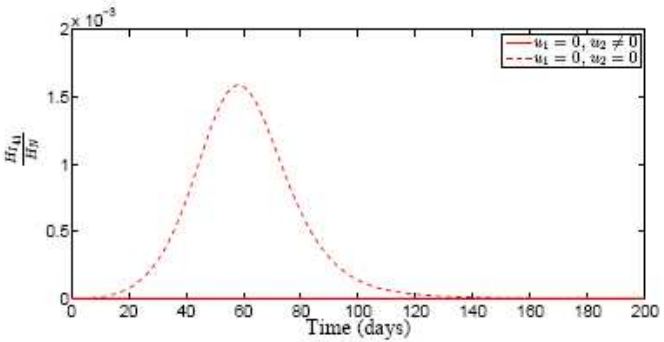

(d) Infected Humans by DEN-4, then by DEN-1

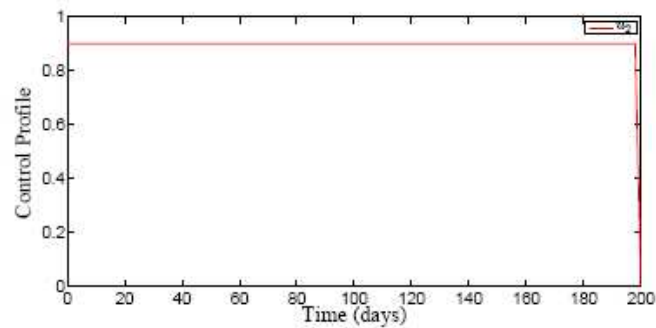

(f) Optimal Control Profile for $u_{2}$

Figure 2: Simulations of System (28) Displaying the Impact of $u_{2}$ (Adulticide) on Dengue Spread with Coexistence of DEN-1 and DEN-4 for Scenario 1

Figure 2 presents the significant impacts of control $u_{2}$ on the dynamical behaviours of the size of infected humans and the total mosquito population. Figures $2 \mathrm{a}$ and $2 \mathrm{~d}$ reveal that the presence of control $u_{2}$ diminishes the numbers of primary and secondary infected humans by DEN-1 to zero throughout the period of intervention. Also, the sizes of the primary and secondary infected humans by DEN-4 with control decrease more rapidly than the situation without control as shown in Figures $2 \mathrm{~b}$ and $2 \mathrm{c}$ These results are in line with the results obtained in Rocha et al. [4]. Although, the authors did not consider the optimal strategy for distributing adulticide control $\left(u_{2}\right)$, but their study of the efficacy of $u_{2}$ on the dynamics of dengue disease spread with the coexistence of two virus serotypes showed that the use of $u_{2}$ is significant to decrease the numbers of primary and secondary infected humans. It is shown in Figure 2e that the size of the mosquito population with control decreased by $80 \%$ at the end of the intervention against the case of no control. Control $u_{2}$ takes effect from the second day of its administration, and is expected to be sustained maximally until time $t=197$ days before dropping sharply to the minimum value at the final time (see Figure $2 \mathrm{f}$ ).

The impact of combined Dengvaxia vaccine and adulticide controls on the dynamical 


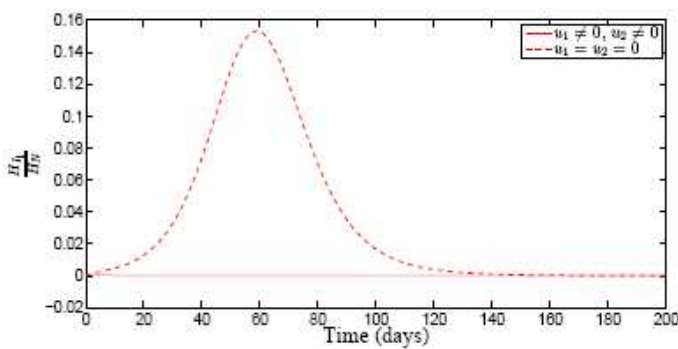

(a) Infected Humans by DEN-1

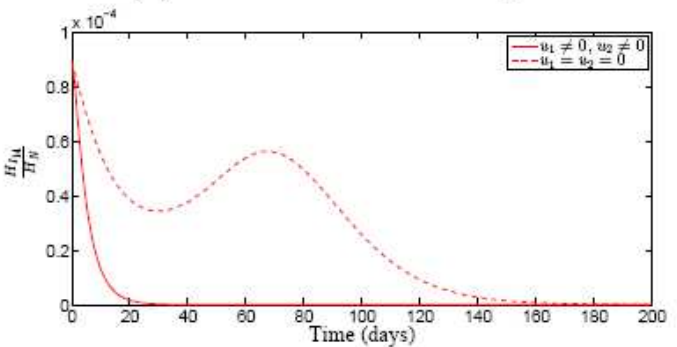

(c) Infected Humans by DEN-1, then by DEN-4

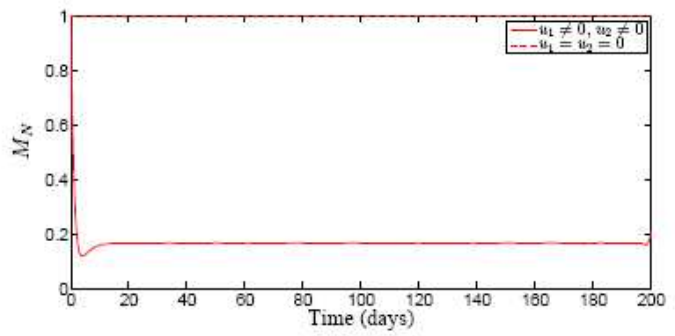

(e) Total Mosquito Population

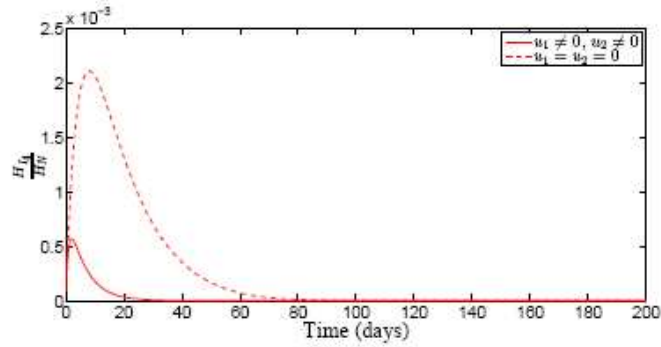

(b) Infected Humans by DEN-4

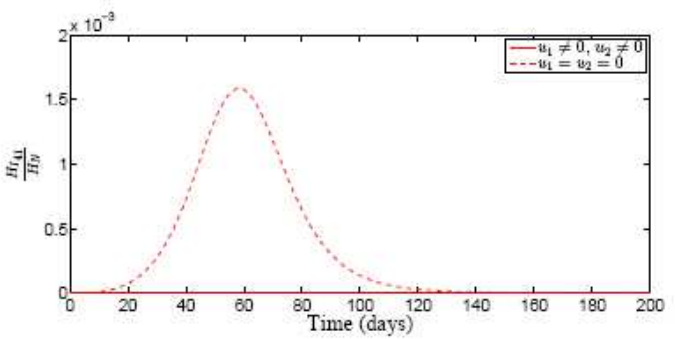

(d) Infected Humans by DEN-4, then by DEN-1

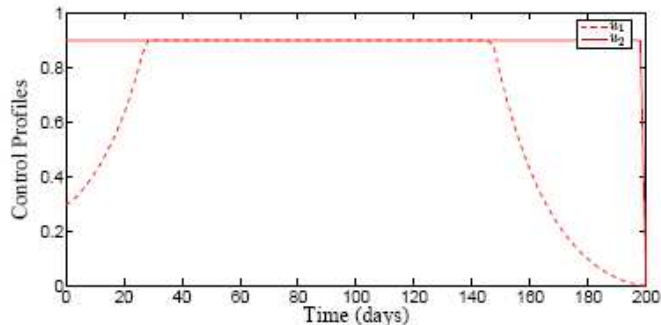

(f) Optimal Control Profiles for $u_{1}$ and $u_{2}$

Figure 3: Simulations of System (28) Displaying the Impacts of Combined Controls $u_{1}$ (Dengvaxia Vaccine) and $u_{2}$ (Adulticide) on Dengue Spread with Coexistence of DEN-1 and DEN-4 for Scenario 1

transmission of dengue is illustrated in Figure 3. It can be seen from Figures 3a-3e that the numbers of primary and secondary infected humans, and the total mosquito population with controls stay near zero than in the case of no control. These results are corroborated by the results of the study of optimal vaccination and adulticide for controlling a single strain dengue transmission in Agusto and Khan [19]. It is revealed by Figure 3f that controls $u_{1}$ and $u_{2}$ must be sustained at the maximum value for 121 and 197 days, respectively, before decreasing to the minimum value at the final time.

Numerical experimentation is carried out on the optimality system for Scenario 2 as well. The graphical results associated with Scenario 2 are omitted here because they are similar to those obtained for Scenario 1. The numbers of primary and secondary infected humans with control $u_{1}$ diminish sharply near zero than in the case of no control when DEN-1 and DEN-2/3 virus co-circulate. It is also observed that mosquito population, in case of control, is constant throughout the period of intervention. The $\mathrm{OC} u_{1}$ is sustained at the upper bound from time $t=12$ days to time $t=126$ days before dropping slowly to the lower bound at time $t_{f}$. 
A huge impact of control $u_{2}$ on the numbers of primary and secondary infected humans by DEN-1 and DEN-2/3, and the total mosquito population is depicted. It is revealed that the numbers of primary and secondary infected humans by DEN- 1 and DEN-2/3 in the presence of control remained close to zero from time $t=20$ days till final time $t=200$ days. This is consistent with the result of the efficacy of $u_{2}$ on the dynamics of dengue disease spread with the coexistence of DEN-1 and DEN-2/3 obtained in Rocha et al. [4]. In addition, the size of mosquito population with control remained near zero from the 3rd day till the end of intervention. It is also found that the $\mathrm{OC} u_{2}$ should be maximally sustained for 197 days before dropping sharply to the lower bound in final time.

The result of combined effort of controls $u_{1}$ and $u_{2}$ reveal that the numbers of primary and secondary infected humans by DEN- 1 and DEN-2/3 in case of control diminish to zero after about 20 days, and remained there till final time $t=200$ days. Furthermore, the size of mosquito population with control declined by $81 \%$ at time $t=8$ days and remained steady until time $t=197$ days. The OC profiles for this strategy reveal that OC $u_{1}$ should be sustained at the upper bound between the time $t=16$ days and $t=140$ days before reducing slowly to the lower bound in time $t_{f}$, while $u_{2}$ should be maximally sustained for 197 days and then drop sharply to lower bound in final time.

Furthermore, when there is no any intervention, the $\mathcal{R}_{0}$ values for this dengue epidemic in Madeira Island for Scenarios 1 and 2, respectively, are approximately $\mathcal{R}_{0}=$ $\max \{2.0889,1.7671\}=2.0889>1$ and $\mathcal{R}_{0}=\max \{2.0908,3.1292\}=3.1292>1$. Similar results were reported in other studies on dengue $[14,18]$. Epidemiologically, these results indicate that the two co-circulating dengue virus strains persist in the population in both scenarios. Also, virus serotype 1 is predominant in Scenario 1, whereas in the case of Scenario 2 , virus serotype $j=2 / 3$ predominates.

\section{Conclusion}

This study has proposed and analyzed a two-strain compartmental model for the dynamics of transmission and control of dengue disease spread if two virus serotypes coexist in Madeira Island. The work is divided into two parts. In the first part, two control parameters $u_{1}$ and $u_{2}$ representing Dengvaxia vaccine and adulticide, respectively, were incorporated into the model. The global asymptotic stability of the model with respect to the DFE and BE points were established. It was showed that the DFE is GAS when both $\mathcal{R}_{01}, \mathcal{R}_{0 j} \leq 1$, and the BE points are GAS if the associated basic reproduction number is above unity.

In the other part, the controls $u_{1}$ and $u_{2}$ were considered as time-dependent variables, and incorporated into the model in order to examine the OC strategy for dengue transmission. We employed PMP to analyse the model. Numerical simulation was performed on the optimality system by adopting three control strategies: use of Dengvaxia vaccine only, application of adulticide only, and combination of Dengvaxia and adulticide. It was found that a strategy, which is made up of Dengvaxia vaccine and adulticide is optimal for the minimization of the spread of dengue and the costs associated with the control implementation for both less and more aggressive cases in Madeira Island. These findings can be helpful to the concerned Madeira Island authorities in bringing the disease under control, if a new outbreak with different virus serotype surfaces in the Island. 


\section{Acknowledgement}

Abidemi is grateful to Universiti Teknologi Malaysia for the benefit of the International Doctoral Fellowship (IDF) in the form of partial school fees waiver and the Federal Government of Nigeria for the opportunity of its financial intervention.

\section{References}

[1] Esteva, L. and Vargas, C. Coexistence of different serotypes of dengue virus. Journal of Mathematical Biology. 2003. 46(1): 31-47.

[2] Phaijoo, G. R. and Gurung, D. B. Modeling impact of temperature and human movement on the persistence of dengue disease. Computational and Mathematical Methods in Medicine. 2017. 2017.

[3] World Health Organization. Dengue and severe dengue. 2018. http://www. who.int/ mediacentre/factsheets/fs117/en/, Accessed 2 January 2019.

[4] Rocha, F. P., Rodrigues, H. S., Monteiro, M. T. T. and Torres, D. F. Coexistence of two dengue virus serotypes and forecasting for Madeira Island. Operations Research for Health Care. 2015. 7: 122-131.

[5] WHO. Dengue Haemorrhagic Fever: Diagnosis, Treatment, Prevention and Control. 2nd Edition. World Health Organization. 1997.

[6] CDC. Dengue. http://www.cdc.gov/dengue/faqFacts/fact.html, Accessed 10 February 2019.

[7] Esteva, L. and Vargas, C. Analysis of a dengue disease transmission model. Mathematical Biosciences. 1998. 150(2): 131-151.

[8] Sungchasit, R. and Pongsumpun, P. Numerical analysis of the transmission model of dengue on SEIR model. International Journal of Bioscience, Biochemistry and Bioinformatics. 2017. 7(2): 93-101.

[9] Rodrigues, H. S., Monteiro, M. T. T. and Torres, D. F. Vaccination models and optimal control strategies to dengue. Mathematical Biosciences. 2014. 247: 1-12.

[10] Rodrigues, H. S., Monteiro, M. T. T., Torres, D. F. and Zinober, A. Dengue disease, basic reproduction number and control. International Journal of Computer Mathematics. 2012. 89(3): 334-346.

[11] Rodrigues, H. S., Monteiro, M. T. T., Torres, D. F. M., Silva, A. C., Sousa, C. and Conceição, C. Dengue in Madeira Island. In Dynamics, Games and Science. Springer. 593-605. 2015.

[12] Boccia, T., Burattini, M., Coutinho, F. and Massad, E. Will people change their vectorcontrol practices in the presence of an imperfect dengue vaccine? Epidemiology and Infection. 2014. 142(3): 625-633.

[13] James, A. Coexistence of two serotypes of dengue virus with and without seasonal variation. Technical Report. 2013.

[14] Mishra, A. and Gakkhar, S. Modeling of dengue with impact of asymptomatic infection and ADE factor. Differential Equations and Dynamical Systems. 2018: 1-17. 
[15] Anggriani, N., Tasman, H., Ndii, M. Z., Supriatna, A. K., Soewono, E. and Siregar, E. The effect of reinfection with the same serotype on dengue transmission dynamics. Applied Mathematics and Computation. 2019. 349: 62-80.

[16] Iboi, E. A. and Gumel, A. B. Mathematical assessment of the role of Dengvaxia vaccine on the transmission dynamics of dengue serotypes. Mathematical Biosciences. 2018. 304: $25-47$.

[17] Morales, N. G., Núñez-López, M., Ramos-Castañeda, J. and Velasco-Hernández, J. Transmission dynamics of two dengue serotypes with vaccination scenarios. Mathematical Biosciences. 2017. 287: 54-71.

[18] Mishra, A. and Gakkhar, S. The effects of awareness and vector control on two strains dengue dynamics. Applied Mathematics and Computation. 2014. 246: 159-167.

[19] Agusto, F. and Khan, M. Optimal control strategies for dengue transmission in pakistan. Mathematical Biosciences. 2018. 305: 102-121.

[20] Pongsumpun, P., Tang, I.-M. and Wongvanich, N. Optimal control of the dengue dynamical transmission with vertical transmission. Advances in Difference Equations. 2019. 2019(1): 176.

[21] Rodrigues, H. S., Monteiro, M. T. T. and Torres, D. F. Seasonality effects on dengue: basic reproduction number, sensitivity analysis and optimal control. Mathematical Methods in the Applied Sciences. 2016. 39(16): 4671-4679.

[22] Buonomo, B. and Della Marca, R. Optimal bed net use for a dengue disease model with mosquito seasonal pattern. Mathematical Methods in the Applied Sciences. 2018. 41(2): $573-592$.

[23] Chan, M. and Johansson, M. A. The incubation periods of dengue viruses. PloS One. 2012. 7(11): e50972.

[24] van den Driessche, P. and Watmough, J. Reproduction numbers and sub-threshold endemic equilibria for compartmental models of disease transmission. Mathematical Biosciences. 2002. 180(1): 29-48.

[25] LaSalle, J. P. The stability of dynamical systems. vol. 25. SIAM. 1976.

[26] Smith, H. L. and Waltman, P. The theory of the chemostat: dynamics of microbial competition. vol. 13. Cambridge University Press. 1995.

[27] Olaniyi, S., Okosun, K. O., Adesanya, S. O. and Areo, E. A. Global stability and optimal control analysis of malaria dynamics in the presence of human travelers. The Open Infectious Diseases Journal. 2018. 10(1): 166-186.

[28] Khan, M. A., Islam, S., Valverde, J. C. and Khan, S. A. Control strategies of hepatitis B with three control variables. Journal of Biological Systems. 2018. 26(01): 1-21.

[29] Pontryagin, L., Boltyanskii, V., Gamkrelidze, R. and Mishchenko, E. The Mathematical Theory of Optimal Processes. Interscience, New York. 1962.

[30] Lenhart, S. and Workman, J. T. Optimal Control Applied to Biological Models. CRC Press. 2007. 